\title{
Diabody Pretargeting with Click Chemistry In Vivo
}

\author{
Sander M.J. van Duijnhoven ${ }^{1}$, Raffaella Rossin ${ }^{1,2}$, Sandra M. van den Bosch ${ }^{3}$, Michael P. Wheatcroft ${ }^{4}$, Peter J. Hudson ${ }^{4}$, \\ and Marc S. Robillard ${ }^{1}$

\begin{abstract}
${ }^{I}$ Tagworks Pharmaceuticals, Eindhoven, The Netherlands; ${ }^{2}$ Oncology Solutions, Philips Research, Eindhoven, The Netherlands; ${ }^{3}$ Precision and Decentralized Diagnostics, Philips Research, Eindhoven, The Netherlands; and ${ }^{4}$ Avipep Pty. Ltd., Melbourne, Victoria, Australia
\end{abstract}

Radioimmunotherapy and nuclear imaging (immuno-PET/SPECT) of cancer with radiometal-labeled antibody fragments or peptides is hampered by low tumor-to-kidney ratios because of high renal radiometal retention. Therefore, we developed and evaluated a pretargeting strategy using click chemistry in vivo to reduce kidney uptake and avoid unwanted radiation toxicity. We focused on the bioorthogonal reaction between a trans-cyclooctene (TCO)-functionalized TAG72 targeting diabody, AVP04-07, and a low-molecular-weight radiolabeled tetrazine probe that was previously shown to have low kidney retention and relatively fast renal clearance. Methods: AVP04-07 diabodies were functionalized with TCO tags, and in vitro immunoreactivity toward bovine submaxillary mucin and tetrazine reactivity were assessed. Next, pretargeting biodistribution studies were performed in LS174T tumor-bearing mice with AVP04-07$\mathrm{TCO}(\mathrm{n})$ (where $\mathrm{n}$ indicates the number of TCO groups per diabody) and radiolabeled tetrazine to optimize the TCO modification grade $\left(0,1.8\right.$, or 4.7 TCO groups per diabody) and the ${ }^{177}$ Lu-tetrazine dose $(0.1,1.0$, or $10 \mathrm{Eq}$ with respect to the diabody). Radiolabeled tetrazine was injected at $47 \mathrm{~h}$ after diabody injection, and mice were euthanized $3 \mathrm{~h}$ later. A pretargeting SPECT/CT study with ${ }^{111} \mathrm{In}$ tetrazine was performed with the optimized conditions. Results: Immunoreactivity for native AVP04-07 was similar to that for TCOfunctionalized AVP04-07, and the latter reacted efficiently with radiolabeled tetrazine in vitro. The combination of the pretargeting component AVP04-07 functionalized with 4.7 TCO groups and 1 Eq of ${ }^{177} \mathrm{Lu}$-tetrazine with respect to the diabody showed the most promising biodistribution. Specifically, high ${ }^{177} \mathrm{Lu}$-tetrazine tumor uptake (6.9 percentage injected dose/g) was observed with low renal retention, yielding a tumor-to-kidney ratio of 5.7. SPECT/CT imaging confirmed the predominant accumulation of radiolabeled tetrazine in the tumor and low nontumor retention. Conclusion: Pretargeting provides an alternative radioimmunotherapy and nuclear imaging strategy by overcoming the high renal retention of lowmolecular-weight radiometal tumor-homing agents through the separate administration of a tumor-homing agent and a radioactive probe with fast clearance.

Key Words: Diels-Alder; click; pretargeting; diabody

J Nucl Med 2015; 56:1422-1428

DOI: 10.2967/jnumed.115.159145

Received Apr. 10, 2015; revision accepted Jun. 29, 2015.

For correspondence or reprints contact: Marc S. Robillard, Tagworks Pharmaceuticals, High Tech Campus 11, 5656 AE, Eindhoven, The Netherlands.

E-mail: marc.robillard@tagworkspharma.com

Published online Jul. 9, 2015.

COPYRIGHT (C 2015 by the Society of Nuclear Medicine and Molecular Imaging, Inc.
$\mathbf{T}$ arget-specific radiolabeled molecules that show rapid tumor targeting and fast clearance from blood and normal tissues are of great interest for nuclear imaging and radiotherapy of solid tumors. Intact monoclonal antibodies (mAbs) generally exhibit superb tumor uptake and retention, but these properties are offset by the long residence time in blood, which results in low tumor-toblood ratios and radiation dose-limiting side effects in bone marrow (1). Alternatively, low-molecular-weight proteins (with masses of $<60 \mathrm{kDa}$ ) and peptides such as Affibody molecules, Nanobodies, single-chain Fv fragments, and noncovalent stable dimers of singlechain Fv fragments (diabodies) are rapidly cleared from the blood by the kidneys and can provide better tumor-to-nontumor ratios at shorter time intervals than mAbs, albeit usually in combination with lower target uptake (2). However, on glomerular filtration, peptides and antibody fragments are generally reabsorbed by the proximal tubule cells in the kidneys to prevent the loss of valuable amino acids, and this step is followed by lysosomal degradation (3). With regard to radiometal-labeled agents, the catabolic radiometal products have been shown to have prolonged renal entrapment, which typically hampers nuclear imaging and radioimmunotherapy procedures with these agents because of possible nephrotoxicity (4-10). Therefore, strategies that reduce the renal uptake of radiometallabeled low-molecular-weight agents to enable the administration of higher radiation doses with reduced risk for nephrotoxicity remain of great interest.

In recent years, several strategies that reduce the renal uptake of radiometal-labeled low-molecular-weight agents have been developed $(6,11-21)$. One example is the conjugation of polyethylene glycol (PEG) to antibody fragments to increase the molecular weight beyond the renal molecular weight cutoff of $60 \mathrm{kDa}$, resulting in an impressive reduction in kidney uptake $(21,22)$. However, because of the reduction in glomerular filtration, these PEGylated antibody fragments have a relatively long circulation time. Therefore, there is still a need for a way to use peptides or small proteins for radioimaging or therapy without having to resort to cumbersome and case-by-case optimization of the structure and while still retaining the fast clearance of small molecules. Here, we focus on the use of an in vivo pretargeting strategy with minimal perturbation of the parent structure and pharmacokinetics (23).

Pretargeting strategies involve the separate administration of a tumor-binding agent, which binds to a tumor-specific extracellular antigen, and a radionuclide. In the first step, the tumor-binding agent is administered and accumulates in the tumor. On blood clearance of the circulating tumor-binding agent, a radiolabeled probe with fast clearance is administered. The probe efficiently targets the radioactivity to the prelocalized agent in the tumor, and unreacted probe is 
rapidly excreted from the circulation, with low renal retention. We previously showed that antibody pretargeting through in vivo click chemistry results in higher tumor-to-nontumor ratios than conventional radiolabeled mAbs (24). In that study, we used a tumor pretargeting strategy based on the bioorthogonal inverse-electron-demand Diels-Alder reaction between a highly reactive trans-cyclooctene (TCO)-tagged TAG72 binding antibody and radiometal-labeled tetrazine. Because of the long circulation time of antibodies, clearing agents were injected to effectively remove residual antibodies from the blood before radiolabeled tetrazine was administered. Radiolabeled tetrazine displayed high tumor uptake, low kidney retention, and relatively fast excretion from the kidneys over time (24).

We hypothesized that translation of the pretargeting strategy to smaller proteins and peptides might be an attractive approach to overcoming the high kidney radiation dose associated with the renal reabsorption of tumor-homing agents labeled with a radiometal. In comparison with our previous pretargeting work on antibodies with a long circulation time (23-25), the relatively fast clearance of low-molecular-weight agents is expected to result in high tumor-to-blood ratios without the need for clearing agents. Among antibody fragments, diabodies $(\sim 55 \mathrm{kDa})$ are of particular interest because they provide greater avidity than other agents, including monovalent single-chain Fv fragments, and therefore typically show higher tumor uptake $(2,21,22)$. In the present study, we explored the use of a TAG72-binding diabody, AVP04-07 (21), as a model construct for a pretargeting approach in LS174T tumorbearing mice (Fig. 1A). The results of the diabody pretargeting approach were compared with previously published data for a conventional radiometal-labeled TAG72-binding diabody analog that showed high tumor targeting and fast blood clearance but high renal retention $(21)$.

\section{MATERIALS AND METHODS}

All reagents and solvents were obtained from commercial sources (Sigma-Aldrich, Acros, Invitrogen, and Merck) and used without further purification unless stated otherwise. ${ }^{111}$ In-indium chloride, ${ }^{177} \mathrm{Lu}$-lutetium chloride, and sodium ${ }^{125}$ I-iodide solutions were purchased from PerkinElmer. Water was distilled and deionized $(18 \mathrm{M} \Omega \mathrm{cm})$ by

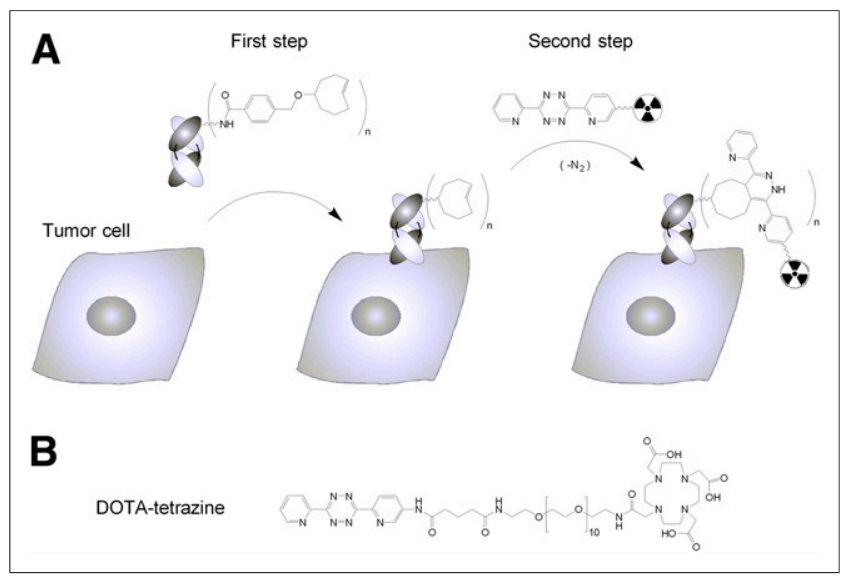

FIGURE 1. (A) Schematic of diabody tumor pretargeting strategy based on inverse-electron-demand Diels-Alder reaction between TCO-tagged diabody and radiolabeled tetrazine. On tumor binding and blood clearance of diabody, administration of radiolabeled tetrazine targets radioactivity to prelocalized diabody in tumor. (B) Molecular structure of DOTA-tetrazine. means of a milli-Q water filtration system (Millipore). The labeling buffers were treated with Chelex-100 resin (BioRad Laboratories) overnight, filtered through $0.22-\mu \mathrm{m}$ filters, and stored at $4^{\circ} \mathrm{C}$. Bolton-Hunter reagent ( $N$-succinimidyl-3-[4-hydroxyphenyl]propionate [SHPP]), Gelcode Blue protein staining solutions, and Zeba desalting spin columns (7-kDa cutoff; $0.5 \mathrm{~mL}$ ) were purchased from Pierce Protein Research (Thermo Fisher Scientific). Mouse serum was purchased from Innovative Research.

The ${ }^{111} \mathrm{In}$ and ${ }^{177} \mathrm{Lu}$ labeling yields for DOTA-tetrazine were determined by radio-thin-layer chromatography (radio-TLC) with ITLCSG strips (Varian Inc.) eluted with $200 \mathrm{mM}$ ethylenediaminetetraacetic acid in saline and imaged on a phosphorimager (FLA-7000; Fujifilm). Under these conditions, free ${ }^{111} \mathrm{In}$ and ${ }^{177} \mathrm{Lu}$ migrated with an $\mathrm{R}_{\mathrm{f}}$ value of 0.9 , whereas ${ }^{111} \mathrm{In}$ - or ${ }^{177} \mathrm{Lu}$-tetrazine remained at the origin. The radiochemical purity of ${ }^{111} \mathrm{In}$ - and ${ }^{177} \mathrm{Lu}$-labeled DOTA-tetrazine was determined by radio-high-pressure liquid chromatography (HPLC) on an Agilent 1100 system equipped with a Gabi radioactive detector (Raytest). The samples were loaded on an Agilent Eclipse XDB-C18 column $(4.6 \times 150 \mathrm{~mm}, 5 \mu \mathrm{m})$ and eluted at $1 \mathrm{~mL} / \mathrm{min}$ with a linear gradient of acetonitrile in water containing $0.1 \%$ trifluoroacetic acid ( $2 \mathrm{~min}$ at $10 \%$ acetonitrile followed by an increase to $45 \%$ acetonitrile in $11 \mathrm{~min}$ ). The ultraviolet wavelength was preset at $254 \mathrm{~nm}$. The ${ }^{125} \mathrm{I}-$ diabody labeling yields were determined by radio-TLC with ITLC-SG strips eluted with a 1:1 methanol-ethyl acetate mixture and imaged on a phosphorimager. Under these conditions, free ${ }^{125} \mathrm{I}$-iodide and ${ }^{125} \mathrm{I}$ SHPP migrated with $\mathrm{R}_{\mathrm{f}}$ values of $0.5-0.9$, whereas ${ }^{125}$ I-diabodies remained at the origin. The radiochemical purity of ${ }^{125}{ }^{12}$-AVP04-07 and TCO-conjugated ${ }^{125}$ I-AVP04-07 was determined by size exclusion chromatography (SEC) and sodium dodecyl sulfate-polyacrylamide gel electrophoresis (SDS-PAGE). SEC was performed on an Agilent 1200 system equipped with a Gabi radioactive detector (Raytest). The samples were loaded on a Superdex 200 10/300 column (GE Healthcare) and eluted with $10 \mathrm{mM}$ phosphate buffer $(\mathrm{pH} 7.4)$ at $0.5 \mathrm{~mL} / \mathrm{min}$. The ultraviolet wavelength was preset at 260 and $280 \mathrm{~nm}$. SDS-PAGE was performed on a Phastgel system with 4\%-15\% PAGE gradient gels (GE Healthcare)

\section{Syntheses of Pretargeting Components}

The synthesis of DOTA-tetrazine (Fig. 1B) (24), the synthesis of the axial isomer of TCO-oxymethylbenzamide- $N$-hydroxysuccinimide (26), and AVP04-07 production have been described elsewhere. AVP04-07, a stable, approximately 55-kDa diabody format of monoclonal antibody CC49, was produced by dimerization of single-chain Fv fragments with short (5-residue) linkers in a bacterial periplasm fermentation system $(21,26)$.

\section{Synthesis of AVP04-07-TCO(n)}

AVP04-07 (1 mg; a solution of $6 \mathrm{mg} / \mathrm{mL}$ in phosphate-buffered saline [PBS]) was modified with 2 or $5 \mathrm{M} \mathrm{Eq}$ of the axial isomer of TCO-oxymethylbenzamide-NHS $(10 \mathrm{mg} / \mathrm{mL}$ in dimethyl sulfoxide) in a total volume of $250 \mu \mathrm{L}$ of PBS. The $\mathrm{pH}$ was adjusted to 9 with $1 \mathrm{M}$ sodium carbonate buffer. The reactions were performed under agitation for $30 \mathrm{~min}$ at room temperature in the dark. Subsequently, the obtained AVP04-07-TCO(n) (where $\mathrm{n}$ indicates the number of TCO groups per diabody) was purified twice through Zeba desalting spin columns (preequilibrated with PBS), and the concentration of the obtained solution was measured with NanoDrop (Thermo Scientific). TCO loading was revealed from the reaction yield between AVP04$07-\mathrm{TCO}(\mathrm{n})(10 \mu \mathrm{g}$ in $50 \mu \mathrm{L}$ of PBS $)$ and 3 or $7 \mathrm{Eq}$ of radiolabeled tetrazine (20 min of incubation at $37^{\circ} \mathrm{C}$ and $300 \mathrm{rpm}$ ).

\section{DOTA-Tetrazine Radiolabeling}

DOTA-tetrazine was dissolved $(1.5 \mathrm{mg} / \mathrm{mL} ; 1.17 \mathrm{mM})$ in $0.2 \mathrm{M}$ ammonium acetate buffer ( $\mathrm{pH}$ 7.0) and stored at $-80^{\circ} \mathrm{C}$ before use. An aliquot of DOTA-tetrazine was mixed with a suitable amount of ${ }^{177} \mathrm{LuCl}_{3}$ or ${ }^{111} \mathrm{InCl}_{3}$ in $0.2 \mathrm{M}$ ammonium acetate ( $\mathrm{pH} 5.5$ ) and incubated 
at $60^{\circ} \mathrm{C}$ for $5 \mathrm{~min}$. Next, the labeling mixture was mixed with a $10 \mathrm{mM}$ diethylenetriaminepentaacetic acid solution $(5 \mu \mathrm{L})$ and a gentisic acid solution $(20 \mathrm{mg} / \mathrm{mL})$ in saline at $\mathrm{pH} 6.5$ and incubated for $5 \mathrm{~min}$. The radiochemical yield and radiochemical purity were assessed by radioTLC and radio-HPLC, respectively, after which the labeling mixture was diluted with sterile saline for animal experiments. The specific activity of ${ }^{177} \mathrm{Lu}$-tetrazine used for biodistribution and that of ${ }^{111} \mathrm{In}-$ tetrazine used for SPECT/CT imaging were 0.07-7 MBq/nmol and approximately $60 \mathrm{MBq} / \mathrm{nmol}$, respectively.

\section{AVP04-07-TCO(n) Radiolabeling}

Radioiodination of AVP04-07-TCO(n) diabodies was performed with the Bolton-Hunter method in accordance with manufacturer instructions. In brief, a suitable amount of sodium ${ }^{125}$ I-iodide in $50 \mu \mathrm{L}$ of PBS was mixed with $0.1 \mu \mathrm{g}$ of Bolton-Hunter reagent (SHPP) and $100 \mu \mathrm{g}$ of chloramine-T ( $N$-chloro-4-methylbenzenesulfonamide, sodium salt). The resulting solution was mixed for 10-20 s, after which ${ }^{125} \mathrm{I}$-SHPP was extracted in toluene and the organic solution was blown to dryness under a gentle stream of $\mathrm{N}_{2}$. AVP04-07-TCO(n) $(0.1 \mathrm{mg}$ in $50 \mu \mathrm{L}$ of PBS) was added to the dry ${ }^{125} \mathrm{I}$-SHPP, the $\mathrm{pH}$ was adjusted to 9 with $1 \mathrm{M}$ sodium carbonate buffer, and the reaction mixture was incubated at room temperature for 30-60 min with gentle shaking. After incubation, the labeling yields were determined by radio-TLC. The ${ }^{125} \mathrm{I}-$ labeled diabodies were purified twice through Zeba spin desalting columns (preequilibrated with saline) in accordance with manufacturer instructions, and the radiochemical purity was determined by radioTLC, SEC, and SDS-PAGE. For animal experiments, the specific activity of ${ }^{125}$ I-AVP04-07-TCO(n) was adjusted to $12-15 \mathrm{kBq} / \mu \mathrm{g}$ by the addition of cold diabody and sterile saline.

\section{Immunoreactivity}

${ }^{125}$ I-labeled AVP04-07 and AVP04-07-TCO(n) were reacted with 20 Eq of bovine submaxillary mucin (BSM, a surrogate target antigen for human TAG72) in $1 \%$ bovine serum albumin in PBS at $37^{\circ} \mathrm{C}$ for $20 \mathrm{~min}$ at $350 \mathrm{rpm}$. Next, the reaction mixtures were analyzed by SEC to identify and quantify diabodies and diabody-BSM complexes.

\section{Animal Experiments}

All animal experiments were performed in accordance with the principles of laboratory animal care (NIH publication 85-23, revised 1985) and Dutch national law Wet op de Dierproeven (Stb 1985, 336). The in vivo experiments were performed in tumor-bearing nude female BALB/c mice (body weight, 20-25 g; Charles River Laboratories). Human colon cancer cell line LS174T was obtained from the American Type Culture Collection and maintained in Eagle minimal essential medium (Sigma) supplemented with $10 \%$ heat-inactivated fetal calf serum (Gibco), penicillin (100 U/mL), streptomycin $(100 \mu \mathrm{g} / \mathrm{mL})$, and 2 mM GlutaMAX (Gibco). Mice were inoculated subcutaneously with $5 \times 10^{6}$ cells in $100 \mu \mathrm{L}$ of sterile PBS and were used $7-10 \mathrm{~d}$ after tumor inoculation, when the tumors reached a size of approximately $70-200 \mathrm{~mm}^{3}$. At the end of each experiment, the mice were anesthetized and euthanized by cervical dislocation. Blood was withdrawn by heart puncture, and selected organs and tissues were harvested and blotted dry. All samples were weighed and then combined with $1 \mathrm{~mL}$ of PBS. The sample radioactivity was counted in a $\gamma$ counter (Wizard 1480; PerkinElmer) along with standards to determine the percentage injected dose/g (\% ID/g) and the percentage injected dose per organ. The tissues from dual-isotope experiments were measured by use of a dual-isotope protocol (energy windows of 10-80 and 155-380 keV for ${ }^{125} \mathrm{I}$ and ${ }^{177} \mathrm{Lu}$, respectively) with cross-contamination correction.

\section{Blood Clearance and Biodistribution Experiments}

Three groups of 3 LS174T tumor-bearing mice were injected intravenously with ${ }^{125} \mathrm{I}-\mathrm{AVP} 04-07,{ }^{125} \mathrm{I}-\mathrm{AVP} 04-07-\mathrm{TCO}(1.8)$, and ${ }^{125} \mathrm{I}-\mathrm{AVP} 04-07-\mathrm{TCO}(4.7)(35 \mu \mathrm{g} / 100 \mu \mathrm{L} /$ mouse; $\sim 0.2 \mathrm{MBq})$. The mice were serially bled at $5 \mathrm{~min}, 30 \mathrm{~min}, 1 \mathrm{~h}, 2 \mathrm{~h}, 6 \mathrm{~h}$, and $24 \mathrm{~h}$. $\mathrm{Next}$, the mice were injected with $6.7 \mathrm{nmol}$ of ${ }^{177} \mathrm{Lu}$-tetrazine (8.52 $\mu \mathrm{g} / 80 \mu \mathrm{L} /$ mouse; containing $100 \mu \mathrm{g}$ of gentisic acid; $\sim 0.5 \mathrm{MBq}$ ) at $47 \mathrm{~h}$ after diabody injection and euthanized at $3 \mathrm{~h}$ after tetrazine injection, and organs and tissues of interest were harvested. Two groups of 4 other LS174T tumor-bearing mice were injected intravenously with ${ }^{125} \mathrm{I}-\mathrm{AVP} 04-07-\mathrm{TCO}(4.7)(35 \mu \mathrm{g} / 100 \mu \mathrm{L} /$ mouse; $~ 0.2 \mathrm{MBq})$, and $0.67 \mathrm{nmol}$ or $67 \mathrm{pmol}$ of ${ }^{177} \mathrm{Lu}$-tetrazine was administered at $47 \mathrm{~h}$ after diabody injection. The mice were euthanized at $3 \mathrm{~h}$ after tetrazine injection, and organs and tissues of interest were harvested. The radioactivity in blood samples, organs, and tissues was counted. Blood clearance data were fitted with a 2-phase exponential decay function, and the area under the curve was determined. Blood half-lives subsequently were derived with the formula $\ln 2 \times \mathrm{AUC} / \mathrm{C}_{0}$, where AUC is the area under the curve and $\mathrm{C}_{0}$ represents the probe concentration $(\% \mathrm{ID} / \mathrm{g})$ in blood at time 0 .

\section{SPECT/CT Imaging Experiment}

One LS174T tumor-bearing mouse was injected with AVP04-07$\operatorname{TCO}(4.7)(35 \mu \mathrm{g} / 100 \mu \mathrm{L})$ and ${ }^{111}$ In-tetrazine $(0.67 \mathrm{nmol} / 80 \mu \mathrm{L}$; containing $100 \mu \mathrm{g}$ of gentisic acid; $\sim 42 \mathrm{MBq}$ ) after the pretargeting protocol. At approximately $90 \mathrm{~min}$ after tetrazine injection, the mouse was anesthetized with isoflurane and imaged on a dedicated smallanimal SPECT/CT system (NanoSPECT/CT; Bioscan) equipped with 4 detector heads and converging 9-pinhole collimators (pinhole diameter, $1.4 \mathrm{~mm}$ ). First, a CT scan was performed (180 projections; $1,000 \mathrm{~ms} /$ projection; $45-\mathrm{kV}$ peak tube voltage; $177-\mathrm{mA}$ tube current; 35-mm field of view), and then a SPECT scan was performed (24 projections; 120 s/projection; photopeaks for ${ }^{111}$ In set to $171 \mathrm{keV}$ [15\% full width] and $245 \mathrm{keV}$ [ $20 \%$ full width]). At $3 \mathrm{~h}$ after tetrazine injection, the mouse was euthanized by anesthesia overdose, and a high-resolution SPECT/CT scan was performed (360 projections and 2,000 ms/projection for CT; 36 projections and $300 \mathrm{~s} /$ projection for SPECT). The SPECT image was reconstructed with HiSPECTNG (SciVis GMBH) to an isotropic voxel size of $300 \mu \mathrm{m}$. The CT image was reconstructed with InVivoScope (Bioscan) to an isotropic voxel size of $200 \mu \mathrm{m}$.

\section{Data Analysis}

The data for $\% \mathrm{ID} / \mathrm{g}$ or percentage injected dose per organ are presented as mean $\pm 1 \mathrm{SD}$. Standard 1-way ANOVAs with Bonferroni post hoc testing for multiple group comparisons, curve fitting, and calculation of the area under the curve were performed with GraphPad Prism version 4.1. The difference between 2 data points was considered statistically significant when the $P$ value was less than 0.05 .

\section{RESULTS}

\section{Diabody Functionalization, Radiochemistry, and In Vitro Characterization}

The TAG72-targeting diabody AVP04-07 was reacted with TCO-oxymethylbenzamide-NHS (26) through lysine residue conjugation (Supplemental Fig. 1) (supplemental materials are available at http://jnm.snmjournals.org) with the aim of affording 2 distinct modification ratios within the range of approximately 2-5 TCO groups per diabody. Radioiodination of AVP04-07 and AVP0407-TCO was performed with the Bolton-Hunter reagent in PBS. The procedure produced a $65 \%-70 \%$ labeling yield and, after size exclusion purification, the radiolabeled diabodies had a radiochemical purity of greater than $98 \%$, as confirmed by radio-TLC and SDS-PAGE analysis (Supplemental Figs. 2 and 3). DOTA-tetrazine was labeled with ${ }^{177} \mathrm{Lu}$ for in vitro reactivity assays and biodistribution experiments or ${ }^{111}$ In for SPECT/CT imaging, with a labeling yield greater than $99 \%$ and a radiochemical purity greater than $95 \%$ 
(Supplemental Figs. 3 and 4). The reaction yield for AVP04-07$\mathrm{TCO}(\mathrm{n})$ and 3 or $7 \mathrm{Eq}$ of radiolabeled tetrazine indicated the presence of, on average, 1.8 or 4.7 TCO groups per diabody, respectively. In an immunoreactivity assay, iodinated AVP04-07-TCO showed quantitative binding to $20 \mathrm{Eq}$ of TAG72-positive BSM (Supplemental Fig. 5).

\section{Optimization of Diabody TCO Loading for In Vivo Pretargeting Experiments}

To determine the optimal diabody TCO loading, we injected 3 ${ }^{125}$ I-labeled AVP04-07 constructs, containing 0, 1.8, and 4.7 TCO groups per diabody, into LS174T tumor-bearing mice. Blood clearance data showed 1.7- and 1.9-fold-slower blood clearance for ${ }^{125} \mathrm{I}$ AVP04-07-TCO(1.8) and ${ }^{125} \mathrm{I}-\mathrm{AVP} 04-07-\mathrm{TCO}(4.7)$, respectively, than for native ${ }^{125}$ I-AVP04-07 (Fig. 2). All diabodies were essentially cleared from the circulation at $50 \mathrm{~h}$ after diabody injection. At $47 \mathrm{~h}$ after diabody injection, $10 \mathrm{Eq}$ of ${ }^{177} \mathrm{Lu}$-tetrazine (with respect to the diabody) were administered by intravenous injection. The dual-isotope biodistribution results at $3 \mathrm{~h}$ after ${ }^{177} \mathrm{Lu}$-tetrazine injection, corresponding to $50 \mathrm{~h}$ after diabody injection, were similar for the 3 diabodies (Fig. 3A; Supplemental Table 1). Specifically, the ${ }^{125} \mathrm{I}$-diabodies showed high tumor uptake $(12-14 \% \mathrm{ID} / \mathrm{g})$, whereas normal tissues and organs showed low to modest levels ( $\sim 0.05 \% \mathrm{ID} / \mathrm{g}$ for muscle, $\sim 0.8 \% \mathrm{ID} / \mathrm{g}$ for spleen, and $2.2-2.9 \% \mathrm{ID} / \mathrm{g}$ for liver). The low ${ }^{125}$ I levels in the stomach and thyroid indicated no significant in vivo dehalogenation of the ${ }^{125}$ I-AVP04-07 constructs.

The administration of $10 \mathrm{Eq}$ of ${ }^{177} \mathrm{Lu}$-tetrazine led to low tumor uptake for mice pretargeted with native AVP04-07, whereas with AVP04-07-TCO(1.8) and AVP04-07-TCO(4.7), the ${ }^{177} \mathrm{Lu}$-tetrazine tumor levels were significantly higher and correlated with the TCO concentration $(0.78 \pm 0.20$ and $1.55 \pm 0.22 \% \mathrm{ID} / \mathrm{g}$, respectively) (Supplemental Table 1). Calculation of the on-tumor reaction yields (based on TCO) for the ${ }^{125}$ I-labeled diabody-TCO constructs and ${ }^{177} \mathrm{Lu}$-tetrazine revealed values of $31.2 \% \pm 7.2 \%$ and $37.7 \% \pm 3.1 \%$ for AVP04-07-TCO(1.8) and AVP04-07-TCO(4.7), respectively. For all other tissues and organs, the ${ }^{177} \mathrm{Lu}$-tetrazine retention was low to very low and independent of the preadministered diabody. Because the tumor-to-nontumor ratios for ${ }^{177} \mathrm{Lu}$-tetrazine in mice pretargeted with AVP04-07-TCO(4.7) were significantly

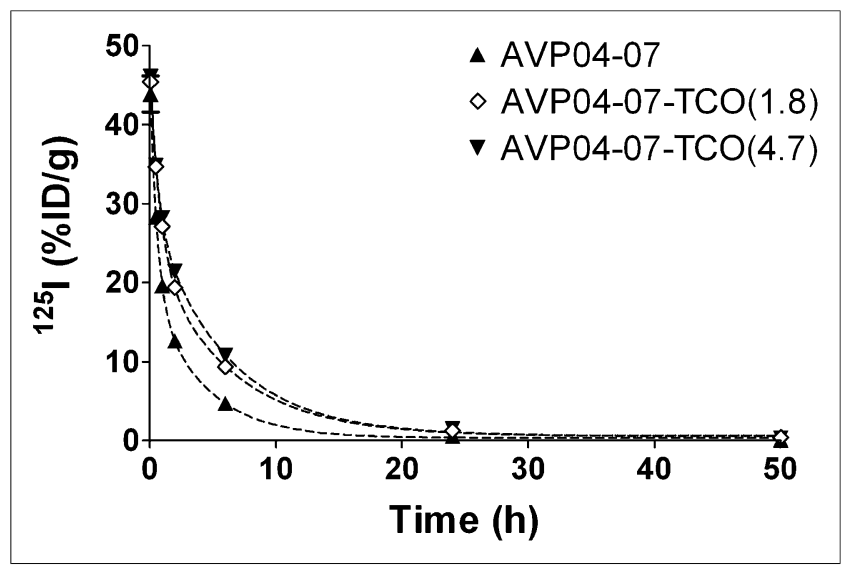

FIGURE 2. Blood clearance of $35 \mu \mathrm{g}$ of ${ }^{125}$ I-AVP04-07, 125I-AVP0407-TCO(1.8), and 125I-AVP04-07-TCO(4.7) in LS174T tumor-bearing mice. Data points represent mean \pm SD \%ID/g $(n=3)$. Half-lives were $1.92 \mathrm{~h}$ for ${ }^{125}$ I-AVP04-07, $3.30 \mathrm{~h}$ for ${ }^{125}$ I-AVP04-07-TCO(1.8), and $3.58 \mathrm{~h}$ for ${ }^{125}$ I-AVP04-07-TCO(4.7).

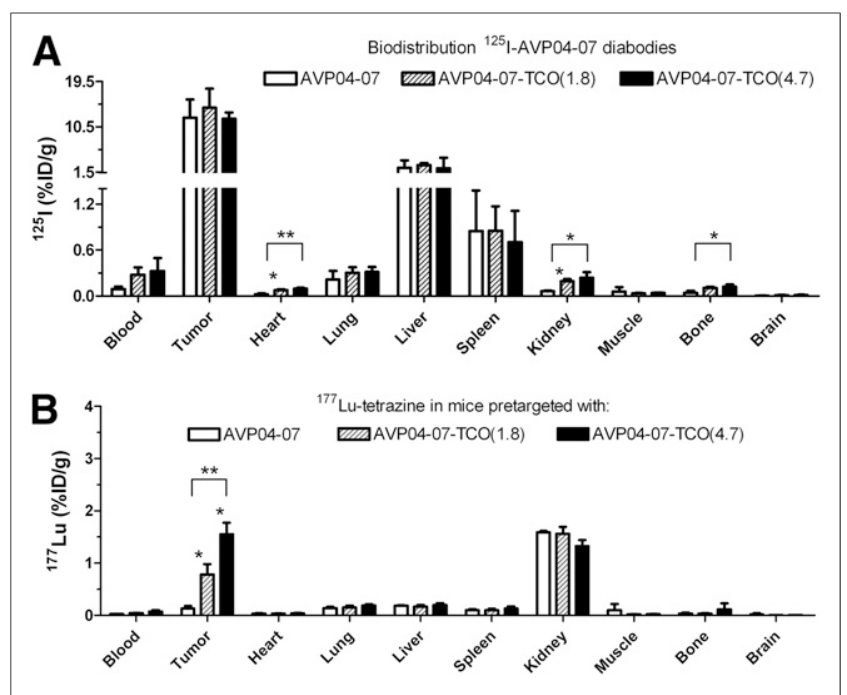

FIGURE 3. Dual-isotope biodistribution for pretargeting with $125 \mathrm{I}-$ AVP04-07, 125I-AVP04-07-TCO(1.8), or 125I-AVP04-07-TCO(4.7) and ${ }^{177} \mathrm{Lu}$-tetrazine in LS174T tumor-bearing mice. Mice were injected with one of the ${ }^{125}$ I-AVP04-07 diabodies, and ${ }^{177}$ Lu-tetrazine (10 Eq, with respect to diabody) was administered at $47 \mathrm{~h}$ after diabody injection. Mice were euthanized $3 \mathrm{~h}$ later. (A) Biodistribution of ${ }^{125}$ I-AVP04-07 diabodies. (B) Biodistribution of ${ }^{177}$ Lu-tetrazine. Bars represent mean \pm SD \% ID/g $(n=3) .{ }^{*} P<0.05 .{ }^{\star \star} P<0.001$.

higher for most tissues than those obtained with AVP04-07-TCO(1.8) (Supplemental Table 2), the former was selected for further evaluation.

\section{Optimization of ${ }^{177} \mathrm{Lu}$-Tetrazine Dose to Improve Tumor Uptake and SPECT/CT Imaging}

To investigate whether tumor uptake and the tumor-to-blood or tumor-to-normal tissue ratios could be further improved, we investigated 2 additional dose levels. Pretargeting biodistribution studies with 1.0 and $0.1 \mathrm{Eq}$ of ${ }^{177} \mathrm{Lu}$-tetrazine (with respect to the diabody) administered at $47 \mathrm{~h}$ after diabody injection were performed in mice pretargeted with ${ }^{125}$ I-AVP04-07-TCO(4.7) (Fig. 4A; Supplemental Table 3). Although the tumor-to-blood ratio decreased with decreasing amounts of injected ${ }^{177}$ Lu-tetrazine (Fig. 4B), most other tumor-to-organ ratios were significantly higher when $1 \mathrm{Eq}$ of ${ }^{177} \mathrm{Lu}-$ tetrazine was administered with respect to AVP04-07-TCO(4.7) than when either 0.1 or $10 \mathrm{Eq}$ were administered (Supplemental Table 4). Specifically, the tumor-to-kidney ratio increased from 1.2 to 5.7 when 1 instead of $10 \mathrm{Eq}$ of ${ }^{177} \mathrm{Lu}$-tetrazine was administered. In addition, tumor uptake was maximal at that dose $(6.9 \pm 1.1 \% \mathrm{ID} / \mathrm{g})$. Therefore, $1 \mathrm{Eq}$ of ${ }^{177} \mathrm{Lu}$-tetrazine (with respect to the diabody) was considered to be the optimal ${ }^{177} \mathrm{Lu}$-tetrazine dose.

A subsequent SPECT/CT imaging experiment with the pretargeting protocol at this optimal dose confirmed high tumor uptake of ${ }^{111}$ In-tetrazine and low retention in nontarget organs (Fig. 5). This finding was expected because we and others previously demonstrated similar biodistributions of ${ }^{177} \mathrm{Lu}$ - and ${ }^{111}$ In-labeled mAbs and low-molecular-weight agents when DOTA was used as a chelator $(25,27-30)$.

\section{DISCUSSION}

The tubular reabsorption and subsequent high renal retention of radiometal-labeled peptides, antibody fragments, and other small proteins hamper their use in nuclear imaging and targeted radiotherapy of cancer because of possible nephrotoxicity. Therefore, 


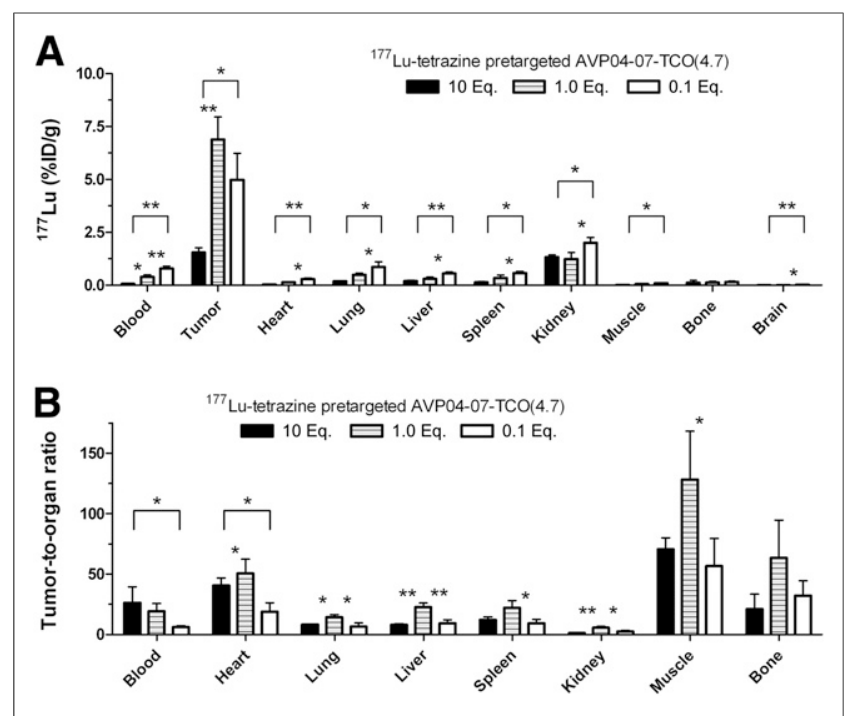

FIGURE 4. ${ }^{177}$ Lu-tetrazine biodistribution in LS174T tumor-bearing mice pretargeted with AVP04-07-TCO(4.7). Mice were injected with AVP04-07-TCO(4.7), and ${ }^{177}$ Lu-tetrazine (10 Eq $[n=3], 1.0 \mathrm{Eq}$, or 0.1 Eq $[n=4]$, with respect to diabody) was administered at $47 \mathrm{~h}$ after diabody injection. Mice were euthanized $3 \mathrm{~h}$ later. (A) Biodistribution of ${ }^{177} \mathrm{Lu}$-tetrazine. (B) Tumor-to-organ ratios for ${ }^{177} \mathrm{Lu}$-tetrazine. Bars represent mean $\pm \mathrm{SD}$. ${ }^{\star} P<0.05 .{ }^{\star \star} P<0.001$.

various strategies that aim to reduce renal uptake of radiometallabeled low-molecular-weight cancer-targeting agents have been developed. Coadministration of cationic amino acids has been shown to inhibit tubular reabsorption of radiolabeled peptides and antibody fragments by up to $35 \%-40 \%(6,11,12)$. Coinfusion of the plasma expander Gelofusine (with or without coadministration of cationic amino acids) also resulted in a strong reduction in kidney uptake for a radiolabeled octreotate (31) and a radiolabeled Nanobody $(28,29)$. In another approach, radiolabeled chelates were designed to be cleaved from the protein or peptide on glomerular filtration but before reabsorption, allowing renal excretion of the radiolabel. This strategy resulted in a $75 \%$ reduction in renal uptake of the radiolabeled chelate (14). Importantly, none of these approaches could completely inhibit the renal reabsorption of small radiolabeled agents and, for the approaches that have been evaluated in tumor-bearing animals, kidney retention was still higher than tumor uptake $(14,32,33)$. Therefore, more efficient strategies for reducing radiation toxicity in the kidneys are needed.

Interestingly, approaches focused on increasing the apparent size of the targeting agents did reduce the glomerular filtration rate and thereby decreased renal reabsorption and strongly improved radionuclide tumor-to-kidney ratios $(15,21)$. For example, both the PEGylation of diabodies and the modification of Affibody molecules with albumin-binding domains resulted in a strong reduction in glomerular filtration and tumor uptake that was 7-fold higher than kidney uptake (in \% ID/g) $(15,21)$. However, these strategies are hampered by the prolonged residence time of the agents in blood, resulting in relatively low tumor-to-blood ratios and the risk of radiation toxicity for bone marrow, similar to that observed with intact antibodies.

We previously developed a click chemistry pretargeting strategy that may be an attractive alternative to reengineering (24). An antiTAG72 diabody, AVP04-07, was selected as a low-molecularweight tumor-homing model construct. Previous work showed that

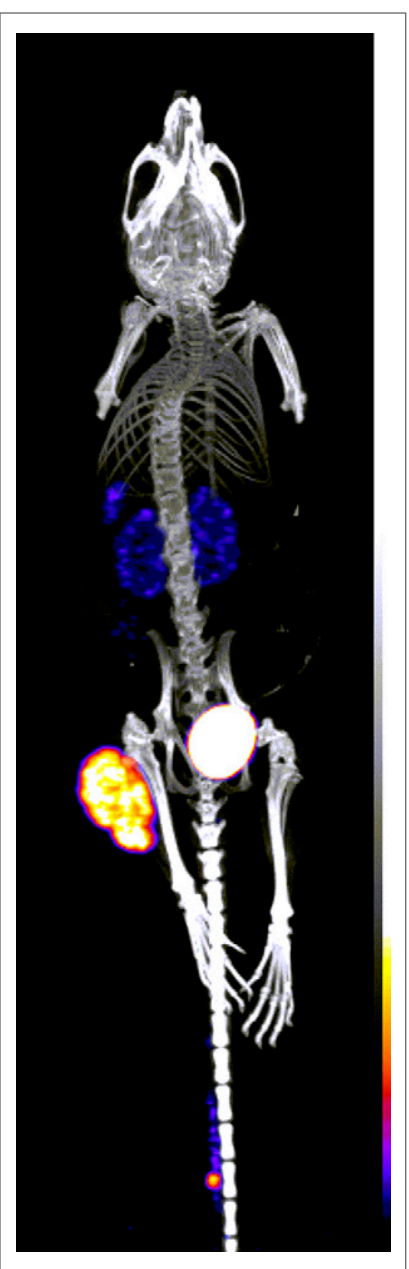

FIGURE 5. SPECT/CT image (maximum-intensity projection) of ${ }^{111}$ In-tetrazine in LS174T tumorbearing mouse pretargeted with AVP04-07-TCO(4.7). Image shows high radioactivity uptake in tumor (hind $\operatorname{limb}$ ) and bladder (urine). Low retention of radioactivity was observed in kidneys, whereas all other organs and tissues showed negligible levels of radioactivity. body. In addition, the diabody-TCO constructs reacted efficiently with the ${ }^{177} \mathrm{Lu}$-radiolabeled tetrazine probe in PBS. In mice, the different radioiodinated diabodies [ $\left.{ }^{125} \mathrm{I}-\mathrm{AVP} 04-07-\mathrm{TCO}(\mathrm{n})\right]$ had comparable biodistribution, indicating that the modification of the diabody with a TCO tag did not significantly perturb its pharmacokinetics.

We observed a relatively minor increase in the blood clearance half-life for the TCO-modified diabodies compared with the native diabody; this increase was most likely due to noncovalent or covalent albumin binding $(34,35)$. The similar tumor uptake for native and tagged diabodies might be explained by a slightly reduced tumor bioavailability for the diabody fraction that is bound to albumin, counteracting the slightly longer blood circulation time and expected increased tumor exposure and binding. After the administration of ${ }^{177} \mathrm{Lu}$-radiolabeled tetrazine and dual-isotope biodistribution analysis, we found on-tumor reaction yields (based 
on TCO) of $31.2 \% \pm 7.2 \%$ and $37.7 \% \pm 3.1 \%$ for AVP04-07$\mathrm{TCO}(1.8)$ and AVP04-07-TCO(4.7), respectively; these values correspond well to the value of $46 \%$ found in an earlier pretargeting study with the same molar dose of full mAb CC49 modified with 8 TCO groups (24). Because ${ }^{177}$ Lu-tetrazine showed the highest tumor accumulation in animals pretargeted with AVP0407-TCO(4.7) and the TCO modification was well tolerated by the diabody in vitro and in vivo, we selected AVP04-07-TCO(4.7) for further evaluation.

We next evaluated blood clearance and biodistribution in LS174T tumor-bearing mice with AVP04-07-TCO(4.7) and different doses of the ${ }^{177} \mathrm{Lu}$-radiolabeled tetrazine probe. We found the highest tumor uptake $(6.9 \% \mathrm{ID} / \mathrm{g})$ and tumor-to-nontumor ratio for $1 \mathrm{Eq}$ of ${ }^{177} \mathrm{Lu}$-tetrazine (with respect to the diabody), with low kidney uptake leading to a tumor-to-kidney ratio of 5.7. The administered TCO-to-tetrazine ratio of 4.7 was in close agreement with those used in other antibody-based pretargeting strategies, in which investigators observed optimal tumor uptake at tag-to-probe ratios of 5-25 for bispecific monoclonal antibodies or Fab fragment combinations that were used to capture radiolabeled bivalent hapten peptides (36-38). Optimization of the dose for AVP04-07$\mathrm{TCO}(4.7)$ and the time interval between the administration of the diabody and the administration of radiolabeled tetrazine may improve the tumor uptake of radiolabeled tetrazine even further.

Pretargeting biodistribution studies revealed a tumor-to-kidney ratio that was more than 20 -fold higher than that previously reported with a radiolabeled DOTA-conjugated AVP04-07 diabody (21), indicating that pretargeting can reduce the kidney radiation dose. Compared with the PEGylation strategy for AVP04-07 (21), the AVP04-07 pretargeting approach resulted in comparable tumorto-kidney ratios (7.0 vs. 5.7) at $48 / 50 \mathrm{~h}$ after diabody injection, with greater than 4-fold better tumor-to-blood ratio for the pretargeting approach, most likely due to differences in the blood clearance rates for TCO-modified AVP04-07 and PEGylated AVP04-07. In a recent dosimetry study, we found that radiolabeled DOTA-tetrazine (or metabolites) was efficiently eliminated from the kidneys over a time course of several days (24). This may further reduce the kidney dose and favor pretargeting in comparison with direct tumor targeting using radiometal-labeled peptides and proteins.

\section{CONCLUSION}

We showed that a pretargeting strategy with a small protein may be an attractive approach for reducing kidney uptake while maintaining high tumor targeting in nuclear imaging and radioimmunotherapy. An effective tumor-targeting agent, an anti-TAG72 diabody that resulted in high kidney uptake when used as a radiometal-labeled analog, showed complete retention of its properties on TCO modification. The administration of radiometal-labeled tetrazine in a second step resulted in efficient tumor uptake and, most importantly, low kidney uptake. This pretargeting strategy could be an important alternative platform, superseding the use of peptides and small proteins as metal-chelate conjugates in imaging and therapy.

\section{DISCLOSURE}

The costs of publication of this article were defrayed in part by the payment of page charges. Therefore, and solely to indicate this fact, this article is hereby marked "advertisement" in accordance with 18 USC section 1734. This research was supported by NanoNextNl (The Netherlands). All authors declare a conflict of interest because of their employment at Tagworks Pharmaceuticals, Philips Research, and Avipep Pty. Ltd. No other potential conflict of interest relevant to this article was reported.

\section{ACKNOWLEDGMENTS}

We thank Dr. Iris Verel (Philips Research), Monique Berben (Philips Research), Caren van Kammen (Maastricht University), and Carlijn van Helvert (Maastricht University) for support with in vivo experiments.

\section{REFERENCES}

1. Boswell CA, Brechbiel MW. Development of radioimmunotherapeutic and diagnostic antibodies: an inside-out view. Nucl Med Biol. 2007;34:757-778

2. Olafsen T, Elgqvist J, Wu AM. Protein targeting constructs in alpha therapy. Curr Radiopharm. 2011;4:197-213.

3. Maack T, Johnson V, Kan ST, Figueiredo J, Sigulem D. Renal filtration, transport, and metabolism of low molecular weight proteins: a review. Kidney Int. 1979;16:251-270.

4. Malmberg J, Sandström M, Wester K, Tolmachev V, Orlova A. Comparative biodistribution of imaging agents for in vivo molecular profiling of disseminated prostate cancer in mice bearing prostate cancer xenografts: focus on ${ }^{111} \mathrm{In}$ - and ${ }^{125}$ I-labeled anti-HER2 humanized monoclonal trastuzumab and ABY-025 Affibody. Nucl Med Biol. 2011;38:1093-1102.

5. Behr TM, Goldenberg DM, Becker W. Reducing the renal uptake of radiolabeled antibody fragments and peptides for diagnosis and therapy: present status, future prospects and limitations. Eur J Nucl Med. 1998;25:201-212.

6. Williams LE, Wu AM, Kenanova VE, Olafsen T, Yazaki PJ. Numerical comparison of iodine-based and indium-based antibody biodistributions. Cancer Biother Radiopharm. 2014;29:91-98.

7. Jin ZH, Furukawa T, Claron M, et al. Positron emission tomography imaging of tumor angiogenesis and monitoring of antiangiogenic efficacy using the novel tetrameric peptide probe ${ }^{64} \mathrm{Cu}$-cyclam-RAFT-c(-RGDfK-) 4 . Angiogenesis. 2012; 15:569-580.

8. Dalmo J, Rudqvist N, Spetz J, et al. Biodistribution of ${ }^{177}$ Lu-octreotate and ${ }^{111} \mathrm{In}$ minigastrin in female nude mice transplanted with human medullary thyroid carcinoma GOT2. Oncol Rep. 2012;27:174-181.

9. de Jong M, Krenning E. New advances in peptide receptor radionuclide therapy. J Nucl Med. 2002;43:617-620.

10. Movahedi K, Schoonooghe S, Laoui D, et al. Nanobody-based targeting of the macrophage mannose receptor for effective in vivo imaging of tumor-associated macrophages. Cancer Res. 2012;72:4165-4177.

11. Rolleman EJ, Valkema R, de Jong M, Kooij PP, Krenning EP. Safe and effective inhibition of renal uptake of radiolabelled octreotide by a combination of lysine and arginine. Eur J Nucl Med Mol Imaging. 2003;30:9-15.

12. Behr TM, Becker WS, Sharkey RM, et al. Reduction of renal uptake of monoclonal antibody fragments by amino acid infusion. J Nucl Med. 1996;37:829-833.

13. Kim I, Kobayashi H, Yoo TM, et al. Lowering of pI by acylation improves the renal uptake of ${ }^{99 \mathrm{~m}} \mathrm{Tc}-$ labeled anti-Tac dsFv: effect of different acylating reagents. Nucl Med Biol. 2002;29:795-801.

14. Li L, Olafsen T, Anderson AL, Wu A, Raubitschek AA, Shively JE. Reduction of kidney uptake in radiometal labeled peptide linkers conjugated to recombinant antibody fragments: site-specific conjugation of DOTA-peptides to a Cys-diabody. Bioconjug Chem. 2002;13:985-995.

15. Orlova A, Jonsson A, Rosik D, et al. Site-specific radiometal labeling and improved biodistribution using ABY-027, a novel HER2-targeting Affibody moleculealbumin-binding domain fusion protein. J Nucl Med. 2013;54:961-968.

16. Li L, Yazaki PJ, Anderson AL, et al. Improved biodistribution and radioimmunoimaging with poly(ethylene glycol)-DOTA-conjugated anti-CEA diabody. Bioconjug Chem. 2006;17:68-76.

17. Vegt E, Eek A, Oyen WJ, de Jong M, Gotthardt M, Boerman OC. Albuminderived peptides efficiently reduce renal uptake of radiolabelled peptides. Eur $J$ Nucl Med Mol Imaging. 2010;37:226-234.

18. Béhé M, Kluge G, Becker W, Gotthardt M, Behr TM. Use of polyglutamic acids to reduce uptake of radiometal-labeled minigastrin in the kidneys. $\mathrm{J} \mathrm{Nucl} \mathrm{Med}$. 2005;46:1012-1015.

19. Rolleman EJ, Krenning EP, van Gameren A, Bernard BF, de Jong M. Uptake of $\left[{ }^{111} \mathrm{In}-\mathrm{DTPA}_{0}\right.$ ] octreotide in the rat kidney is inhibited by colchicine and not by fructose. J Nucl Med. 2004;45:709-713.

20. Gotthardt M, van Eerd-Vismale J, Oyen WJ, et al. Indication for different mechanisms of kidney uptake. J Nucl Med. 2007;48:596-601. 
21. Li L, Turatti F, Crow D, et al. Monodispersed DOTA-PEG-conjugated anti-TAG72 diabody has low kidney uptake and high tumor-to-blood ratios resulting in improved ${ }^{64} \mathrm{Cu}$ PET. J Nucl Med. 2010;51:1139-1146.

22. Li L, Crow D, Turatti F, et al. Site-specific conjugation of monodispersed DOTAPEGn to a thiolated diabody reveals the effect of increasing PEG size on kidney clearance and tumor uptake with improved 64-copper PET imaging [published correction appears in Bioconjug Chem. 2011;22:1256]. Bioconjug Chem. 2011;22: 709-716.

23. Rossin R, Renart Verkerk P, van den Bosch SM, et al. In vivo chemistry for pretargeted tumor imaging in live mice. Angew Chem Int Ed Engl. 2010;49:3375-3378.

24. Rossin R, Läppchen T, van den Bosch SM, Laforest R, Robillard MS. Diels-Alder reaction for tumor pretargeting: in vivo chemistry can boost tumor radiation dose compared to directly labeled antibody. J Nucl Med. 2013;54:1989-1995.

25. Rossin R, van den Bosch SM, ten Hoeve W, et al. Highly reactive trans-cyclooctene tags with improved stability for Diels-Alder chemistry in living systems. Bioconjug Chem. 2013;24:1210-1217.

26. Powers GA, Hudson PJ, Wheatcroft MP. Design and production of multimeric antibody fragments, focused on diabodies with enhanced clinical efficacy. Methods Mol Biol. 2012;907:699-712.

27. Zhang H, Chen J, Waldherr C, et al. Synthesis and evaluation of bombesin derivatives on the basis of pan-bombesin peptides labeled with indium-111, lutetium-177, and yttrium-90 for targeting bombesin receptor-expressing tumors. Cancer Res. 2004;64:6707-6715.

28. Vallabhajosula S, Kuji I, Hamacher KA, et al. Pharmacokinetics and biodistribution of ${ }^{111} \mathrm{In}$ - and ${ }^{177} \mathrm{Lu}$-labeled J591 antibody specific for prostate-specific membrane antigen: prediction of ${ }^{90} \mathrm{Y}-\mathrm{J} 591$ radiation dosimetry based on ${ }^{111} \mathrm{In}$ or ${ }^{177}$ Lu? J Nucl Med. 2005;46:634-641.
29. Mohsin H, Fitzsimmons J, Shelton T, et al. Preparation and biological evaluation of ${ }^{111} \mathrm{In}-,{ }^{177} \mathrm{Lu}-$ and ${ }^{90} \mathrm{Y}$-labeled DOTA analogues conjugated to B72.3. Nucl Med Biol. 2007;34:493-502.

30. Rossin R, van Duijnhoven SM, Läppchen T, van den Bosch SM, Robillard MS. Trans-cyclooctene tag with improved properties for tumor pretargeting with the Diels-Alder reaction. Mol Pharm. 2014;11:3090-3096.

31. Melis M, Bijster M, de Visser M, et al. Dose-response effect of Gelofusine on renal uptake and retention of radiolabelled octreotate in rats with CA20948 tumours. Eur J Nucl Med Mol Imaging. 2009;36:1968-1976.

32. Gainkam LO, Caveliers V, Devoogdt N, et al. Localization mechanism and reduction of renal retention of technetium-99m labeled epidermal growth factor receptor-specific Nanobody in mice. Contrast Media Mol Imaging. 2011;6:85-92.

33. D'Huyvetter M, Vincke C, Xavier C, et al. Targeted radionuclide therapy with a ${ }^{177}$ Lu-labeled anti-HER2 Nanobody. Theranostics. 2014;4:708-720.

34. van den Bosch SM, Rossin R, Renart Verkerk P, et al. Evaluation of strained alkynes for Cu-free click reaction in live mice. Nucl Med Biol. 2013;40:415-423.

35. Poole TH, Reisz JA, Zhao W, Poole LB, Furdui CM, King SB. Strained cycloalkynes as new protein sulfenic acid traps. J Am Chem Soc. 2014;136:61676170 .

36. Sharkey RM, Cardillo TM, Rossi EA, et al. Signal amplification in molecular imaging by pretargeting a multivalent, bispecific antibody. Nat Med. 2005;11:1250-1255.

37. Karacay H, Brard Y, Sharkey RM, et al. Therapeutic advantage of pretargeted radioimmunotherapy using a recombinant bispecific antibody in a human colon cancer xenograft. Clin Cancer Res. 2005;11:7879-7885.

38. van Rij CM, Frielink C, Goldenberg DM, et al. Pretargeted immunoPET of prostate cancer with an anti-TROP- $2 \times$ anti-HSG bispecific antibody in mice with PC3 xenografts. Mol Imaging Biol. 2015;17:94-101. 